\title{
Magnetic excitations in a nanocontact
}

\author{
J. M. D. Coey, ${ }^{1}$ L. Berger, ${ }^{1,2}$ and Y. Labaye ${ }^{1,2}$ \\ ${ }^{1}$ Physics Department, Trinity College, Dublin 2, Ireland \\ ${ }^{2}$ Laboratoire de Physique de l'Etat Condensé, UMR 6087 CNRS, Université du Maine, F-72085 Le Mans, France
}

(Received 5 February 2001; published 21 June 2001)

\begin{abstract}
The domain wall in a ferromagnetic nanocontact adopts a specific configuration-Néel-like, vortex, or Bloch-like-depending on the dipole-dipole interactions governed by the size and shape of the contact and its atomic structure. Spontaneous thermal fluctuations between these modes arise in a soft ferromagnet at room temperature when the dimensions of the contact are less than about $10 \mathrm{~nm}$. The giant magnetoresistance of a nanocontact may be reduced, but not eliminated by the mode fluctuations.
\end{abstract}

DOI: 10.1103/PhysRevB.64.020407 PACS number(s): 75.60.Ch, 75.40.Mg, 75.20. $-\mathrm{g}$, 73.63.Rt

Contacts between ferromagnetic electrodes which have their magnetization directed parallel or antiparallel to each other are the basis of the emerging science of spin electronics. The electrodes may be separated by a thin metallic layer (spin valve) or a thin insulating layer (tunnel junction), or else they may be in direct contact with each other (nanocontact). Much effort is being directed to perfecting spin valves and tunnel junctions as sensors for magnetic recording and as storage elements for magnetic memory. Some nanocontacts show impressive magnetoresistance effects at room temperature, ${ }^{1}$ especially in half-metallic systems, ${ }^{2}$ but little is known of their magnetic structure. It was recently predicted that very narrow domain walls with dimensions comparable to the length of the nanocontact itself should exist, even in soft magnetic materials. ${ }^{3}$ There have been reports of domain walls in ferromagnetic thin films patterned with micron-size constrictions, ${ }^{4,5}$ but the studies of domain walls in nanometer-scale constrictions have been restricted to micromagnetic calculations ${ }^{3}$ and simulations, based on the continuum approximation, ${ }^{6,7}$ or on lattice sums. ${ }^{8}$ Here we point out that these nanowalls are subject to magnetic fluctuations, which may influence the spin polarization of electrons as they traverse the contact.

To illustrate the idea, consider the simple "isthmus", nanocontact illustrated in Fig. 1. A thread of ferromagnetic material of length $l$ and radius $r_{0}$ connects two semi-infinite slabs of the same material. We assume a common anisotropy axis $\mathrm{O} z$ throughout, with anisotropy constant $K$. The atoms are arranged on a square lattice with lattice parameter $a$. Each atom has a classical spin $|\mathbf{S}|=1$ and moment $1 \mu_{B}$. There is nearest-neighbor exchange coupling of strength $J$. The normal domain wall width $\delta_{0}=\pi\left(J S^{2} / a K\right)^{1 / 2}$ is of order $200 \mathrm{~nm}$, taking values typical of a soft ferromagnet $\left(J / k_{B}\right.$ $\left.=100 \mathrm{~K}, a=0.2 \mathrm{~nm}, K=10^{3} \mathrm{~J} \mathrm{~m}^{-3}\right)$. Using the continuum approximation of micromagnetism, Bruno has shown that the wall width $\delta$ in an isthmus with $l \ll \delta_{0}$ is approximately equal to $l{ }^{3}$ Labaye et al. have confirmed this in Monte-Carlo simulations on an atomic lattice. ${ }^{8}$ The total energy is given by the expression

$$
\begin{aligned}
E_{\mathrm{tot}}= & \sum_{i}\left\{(1 / 2) \sum_{j}^{n n} J \mathbf{S}_{i} \mathbf{S}_{j}+K_{i} a^{3} \sin ^{2} \theta_{i}+G \sum_{j \neq i}\left[\mathbf{S}_{i} \mathbf{S}_{j} r_{i j}^{2}\right.\right. \\
& \left.\left.-3\left(\mathbf{S}_{i} r_{i j}\right)\left(\mathbf{S}_{j} r_{i j}\right)\right] / r_{i j}^{5}\right\},
\end{aligned}
$$

where $G=\mu_{0} \mu_{B}^{2} / 4 \pi$ and the sums are over all atomic sites in the nanocontact. The three terms represent the exchange, anisotropy, and dipole-dipole interactions, respectively.

Basically, three types of nanowalls can appear in the isthmus when the two ferromagnetic slabs are oppositely magnetized. These are (i) Bloch-type walls where the magnetization rotates in the $y z$ plane, with $\phi= \pm \pi / 2$, (ii) Néel-type walls where the magnetization rotates in the $z x$ plane, with $\phi=0$ or $\pi$, and (iii) walls with more complicated vortex structures, where $\phi$ is variable within a plane. The magnetization direction in the slabs adjacent to the isthmus will also be perturbed. The lowest-energy state depends on the atomic structure and the dimensions $l, r_{0}$, as well as the values of $J$ and $K$.

For simplicity, we focus on the first two nanowalls, illustrated in Fig. 1. The Bloch-type walls can have positive or negative chirality since the magnetization may rotate clockwise or anticlockwise in the $y z$ plane. Likewise, the magnetization of the Néel walls can rotate in either sense in the $z x$ plane. Either the Bloch-type or the Néel-type wall is lower in energy. The energy difference between them, $\Delta_{\mathrm{BN}}$, is due
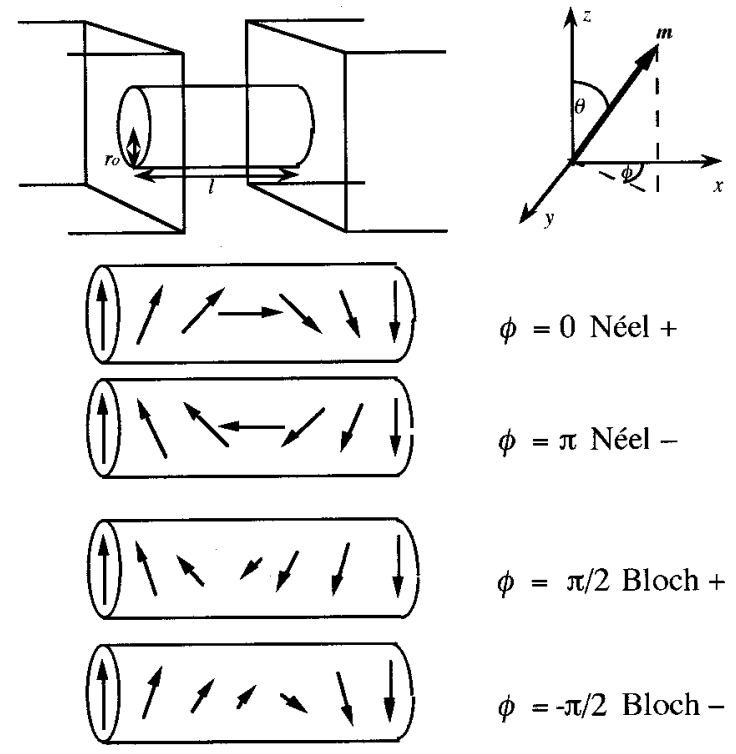

FIG. 1. The "isthmus" nanocontact. Four magnetic modes are shown, two Bloch-type modes with opposite chirality $(\phi= \pm \pi / 2)$, and two Néel-type modes $(\phi=0, \pi)$. 


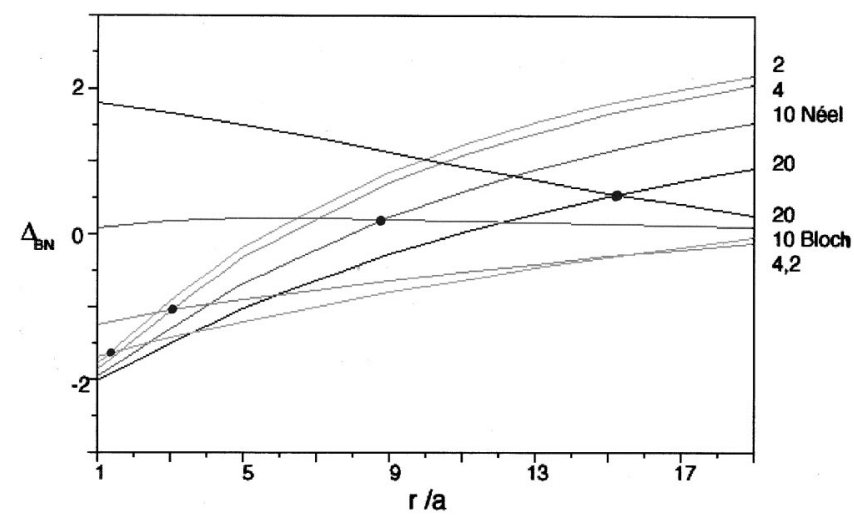

FIG. 2. Energy differences per atom in units of $G / a^{3}$ between the Bloch and Néel modes for nanocontacts of different widths $l / r$ as a function of the radius of the contact in units of $a$. The four curves are for $l=2,4,10$, and $20 a$.

essentially to the dipole-dipole interaction. The contribution of bulk anisotropy to the energy is unimportant for narrow walls in soft ferromagnets, although surface anisotropy $K_{s}$ $\approx 1 \mathrm{~mJ} \mathrm{~m}^{-2}(2.9 \mathrm{~K}$ per surface atom) may influence the nanowall configuration.

Some results of the energy difference $\Delta_{\mathrm{BN}}$ deduced from lattice sums of the total energy with $K=0$ and the constraint $\phi=0$ or $\pi / 2$ are shown in Fig. 2. The order of magnitude of $\Delta_{\mathrm{BN}}$ is $G / a^{3}(0.08 \mathrm{~K})$ per atom in the nanowall. When the number of atoms $n$ in the wall is sufficiently small, $\Delta_{\mathrm{BN}}$ can be of order $k_{B} T$. Spontaneous thermal fluctuations will then occur from one mode to another, as indicated in Figure 3.

Thermal excitations among wall modes will be a feature of any sufficiently small ferromagnetic nanocontact between domains magnetized in different directions. The fluctuations between different nanowall configurations are analogous to superparamagnetic fluctuations between different directions of magnetization of a ferromagnetic nanoparticle. In each case, the relaxation process can be represented by the product of an attempt frequency and the probability of overcoming the energy barrier $\Delta_{b}$ separating the configurations, Hence,

$$
\nu=\nu_{0} \exp \left(-\Delta_{b} / k_{B} T\right) .
$$

In the present case, $\Delta_{b}=\Delta_{\mathrm{BN}}$ because the wall energy varies monotonically with $\phi$. The attempt frequency $\nu_{0}$ is of order the ferromagnetic resonance frequency in the anisotropy and dipole fields, $10^{10} \mathrm{~Hz}$. At room temperature the standard stability condition $\Delta_{b} / k_{B} T \approx 25,{ }^{9}$ yields $n \approx 10^{5}$, corresponding to a size of about $10 \mathrm{~nm}$. Usually a vortex mode lies intermediate in energy between the Bloch and Néel modes, and the size of contact below which spontaneous thermal fluctua-

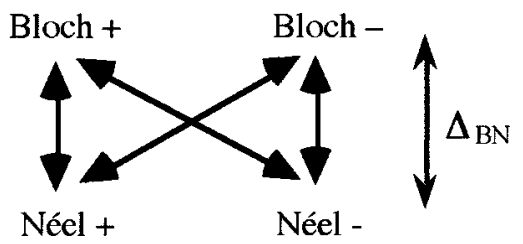

FIG. 3. Mode fluctuations in a ferromagnetic nanocontact. tions occur at room temperature may be a little bigger. Quantum tunneling between different modes is expected to be significant only in very tiny nanocontacts, containing of order 10 atoms. ${ }^{10}$

We now ask what effect the magnetic mode fluctuations can have on electrons passing through the contact. They provide an extra channel for inelastic scattering in a small volume, and there is the possibility that they may induce spin flip. Considering the problem classically, the time spent by a ballistic electron in the nanoconstriction is of order $l / v_{F}$ where $v_{F}$ is the Fermi velocity $\left(\approx 10^{6} \mathrm{~m} \mathrm{~s}^{-1}\right)$; this time is about $10^{-14} \mathrm{~s}$. The spin-polarized conduction electrons are subject to an exchange field which produces a spin splitting $E_{\uparrow \downarrow}$, where $E_{\uparrow \downarrow} / \mathrm{h}$ is approximately $10^{15} \mathrm{~Hz}$. Spin flip would therefore be possible if the mode fluctuations had a transverse component at the Larmor precession frequency of the spin-polarized electron, but it is clear from Eq. (2) that even in the smallest contacts they are considerably slower. The $4 \mathrm{~s}$ electrons in a ferromagnetic metal, which experience an exchange field one or two orders of magnitude less than that for the $3 d$ electrons, also have a Larmor precession frequency which is much faster than the transverse fluctuations of the magnetization; no spin flip is expected for them either. In other terms, domain wall dynamics are governed by a domain-wall velocity which is of order $100 \mathrm{~ms}^{-2},{ }^{11}$ so the time scale for changing the configuration of the contact is of order $10^{-10} \mathrm{~s}$.

A small effect on the resistance is anticipated due to different wall widths for the different modes. For diffusive transport, the wall resistance is predicted to vary as $\delta^{-2}{ }^{12}$ The change in wall resistance due to mode fluctuations may be a few percent.

Many of the atoms in a nanocontact lie at the surface, where the $3 d$ states tend to be localized, especially in halfmetallic ferromagnetic oxides with narrow $d$-bands. The bandwidth of the local density of states $2 \sum_{j}^{n n} t$, where $t$ is the transfer integral, will be reduced to roughly $\frac{2}{3}$ of its bulk value at the surface. Electron transport across the nanocontact is then either by hopping via localized states or by tunneling, or some combination of the two. A hopping electron is subject to spin flip at every hopping site where its wave function is projected along an axis different from $\mathrm{O} z$. The transmission probability $T$ across the wall then depends on the profile $S_{z}(x)$, which is practically the same for Bloch and Néel walls when $G$ is small. In a wall where $d \theta / d x$ is constant, $\delta$ is the wall width and $\nu=\delta / \lambda$, where $\lambda$ is the hopping length, $T \approx\left\{\cos ^{2}(\pi / 2 \nu)\right\}^{v}$. However, the Néel wall in a long isthmus splits into two $90^{\circ}$ half-walls at either end, ${ }^{8}$ and the transmission is then $\left\{\cos ^{2}(\pi / 4)\right\}^{2}$. In this case, thermal excitations decrease the magnetoresistance of the contact by at most a factor of 2 .

There is no tendency to create a $(2 n+1) \pi$ domain wall by thermal fluctuation because the energy cost $\approx J S^{2} r_{0}^{2} / a l$, is greater than $k_{B} T$ for all but the tiniest contacts where $r_{0}, l$ $\approx a$.

Following Eq. (2), the mode fluctuations in a nanocontact could be detected by looking for a thermally activated term in the magnetoresistance, particularly in contacts made from 
half-metallic oxides with hopping conduction or in metals with a large residual resistivity. The nanocontacts themselves, with dimensions of order $10 \mathrm{~nm}$ could be prepared from thin films by focused ion beam milling or electronbeam lithography. Otherwise they might be grown electrochemically. ${ }^{13,14}$ In order to separate the resistivity change due to domain wall mode fluctuations from the intrinsic temperature dependence, a bridge configuration could be used with two contacts of different dimensions. An alternative approach would be to detect the stray field fluctuations from a nanocontact prepared in the sensing coil of a microsquid.

In summary, we propose that a new type of localized mesoscopic magnetic excitation occurs in ferromagnetic nanocontacts. It is illustrated in the simplest, isthmus geometry in terms of thermally excited fluctuations between Bloch-type and Néel-type wall configurations. These excitations are also expected to occur in nanowires and in pinhole contacts in tunnel junctions. The mode excitations may reduce, but not eliminate the giant magnetoresistance. Their effects are likely to be more evident in hopping contacts rather than ballistic or diffusive contacts. It is a challenge to detect these magnetic-mode fluctuations experimentally.

This work was supported by Enterprise Ireland, Contract No. ST/1999/125, and as part of the Ireland-France Exchange Scheme. It also forms part of the EC "Magnoise", project.
${ }^{1}$ N. Garcia, M. Muñoz, and Y-W. Zhao, Phys. Rev. Lett. 82, 2923 (1999).

${ }^{2}$ J. Versluijs, M. A. Bari, and J. M. D. Coey, Phys. Rev. Lett. (to be published June 2001).

${ }^{3}$ P. Bruno, Phys. Rev. Lett. 83, 2425 (1999).

${ }^{4}$ A. Hirohata, Y. B. Xu, C. C. Yao, H. T. Leung, W. Y. Lee, S. M. Gariner, D. G. Hasko, J. A. C. Bland, and S. N. Holmes, J. Appl. Phys. 87, 4727 (2000); J. Magn. Magn. Mater. (to be published).

${ }^{5}$ N. Mathur, P. B. Littlewood, N. K. Todd, S. P. Isaac, B. S. Teo, D. J. Kang, E. J. Tarte, Z. H. Basrber, J. E. Evetts, and M. G. Blamire, J. Appl. Phys. 86, 6287 (1999).

${ }^{6}$ R. P. van Gorkom, S. J. C. H. Theeuwen, K. P. Wellock, N. N. Gribov, J. Caro, and S. Radelaar, J. Appl. Phys. 85, 6196 (1999).
${ }^{7}$ R. D. McMichael, J. Eicke, M. J. Donahue, and D. G. Porter (unpublished).

${ }^{8}$ Y. Labaye, L. Berger, and J. M. D. Coey (unpublished).

${ }^{9}$ A. Aharoni, Theory of Ferromagnetism (Oxford, New York, 1996).

${ }^{10}$ W. Wernsdorfer, E. B. Orozco, B. Barbara, A. Benoit, D. Mailly, N. Demoncy, H. Pascard, O. Kubo, and H. Nakano, IEEE Trans. Magn. 34, 973 (1998).

${ }^{11}$ A. Hubert and R Schäfer, Magnetic Domains (Springer, Berlin, 1998).

${ }^{12}$ P. M. Levy and S. Zhang, Phys. Rev. Lett. 79, 5110 (1997).

${ }^{13}$ A. F. Morpurgro, C. M. Marcus, and D. P. Robinson, Appl. Phys. Lett. 74, 2084 (1999).

${ }^{14}$ N. García, I. G. Savaliev, Y-W. Zhao, and A. Zlatkine, J. Magn. Magn. Mater. 214, 712 (2000). 